\title{
The effect of tooth shape on the breakdown of insects
}

\author{
A. R. Evans and G. D. Sanson \\ Department of Biological Sciences, Monash University, Clayton, Victoria 3168, Australia \\ (Accepted 7 May 1998)
}

\begin{abstract}
This study investigates the effect of tooth sharpness on the force and energy necessary for a tooth to divide foods of differing physical properties. The material properties of foods are likely to affect the shape that is best to divide them. In order to test the effect of sharpness on a tooth's proficiency in dividing insect prey, experiments involving penetrating and driving through insect prey of differing physical properties were carried out using specially designed punches that varied in tip- and cusp-sharpness. A refinement of tooth measurements which are relevant to their effectiveness in penetrating and driving through food is expounded. 'Tip-sharpness' is defined as the radius of curvature at the tip of a cusp. 'Cusp-sharpness' is defined as the volume or surface area of the tooth per unit distance from the tip of a cusp. The experiments show that tooth shape is important in the force and energy needed for teeth to penetrate and drive through insects. Also, the scale of teeth is important - teeth of the same shape but different size vary in their effectiveness to divide foods.
\end{abstract}

Key words: tooth sharpness, tooth function, physical properties, scale, insectivory

\section{INTRODUCTION}

An important advance in recent years in the interpretation of the function of mammalian teeth from their morphology has been the recognition of finer divisions in the traditional dietary category of insectivory. Insects and other invertebrates have been shown to exhibit a wide range of physical characteristics (Hepburn \& Chandler, 1976; Freeman, 1979, 1981; Hill, 1985; Strait, 1991), but previously the diversity of physical properties of insects had not been related to tooth function. Since certain insectivorous species have been found to exploit only sections of the invertebrate prey available (e.g. Ross, 1967; Black, 1974; Freeman, 1979; Warner, 1985), it has been thought that this choice of specific insects could be reflected in their dental morphology. Freeman's $(1979,1981)$ work on tooth and jaw morphology in bats taking hard- and soft-shelled insect prey showed that such differences in dentition do exist, while the investigations of Strait $(1991,1993)$ have expanded the knowledge of the dental differences between various guilds which were all once regarded as 'insectivorous'.

Another advance in these studies was made by Lucas (1979) in his application of fracture mechanics to food items in order to explain how differences in food texture could affect gross dental morphology. Lucas \& Luke (1984) expanded on this work to propose three food categories for primates based on physical properties, and then hypothesized the tooth shapes that would maximize breakdown of each of these, require the least time, and minimize potential tooth damage. They suggested that 'tough and/or soft foods' (Lucas \& Luke, 1984: 288) are most efficiently divided by sharp bladelike cusps. According to Griffith's theory of crackpropagation (Gordon, 1968), cracks are not self-propagating in these materials and so must be driven through the material by blades.

Previously, several aspects of tooth form have been interpreted as being the most important in the breakdown of prey such as insects. Strong or stiff foods are most efficiently broken down by minimizing contact area between the tooth and the food. Such a concentration of jaw forces onto a small area maximizes the pressure on the food for a given force. Lucas (1982) considered that radius of curvature of tooth tip would be most important in penetrating foods, as it will be proportional to the surface area of contact for a given applied load. Therefore, teeth with a smaller radius of curvature will require less force to penetrate foods.

Strait $(1991,1993)$ attempted to give a functional interpretation to Kay \& Hiiemae's (1974) 'shearing ratio', i.e. the sum of six crest lengths on the molars scaled to a factor such as body mass, tooth length or tooth area. For foods that require both blades and a small surface area of contact, Strait hypothesized that decreasing the blade length will decrease the surface area of contact and so increase the pressure, i.e. that blade length $\propto$ surface area of contact. She equated 
blades to the shearing crests on teeth, and so deduced that animals which eat strong or stiff foods that require blades should have short shearing crests in order to minimize the area of contact. As weak and pliant prey do not require high stresses to yield or fracture, critical stress concentrations can be achieved with a relatively larger area of contact. From this she predicted that the teeth of soft-object insectivores will have relatively long shearing features. Long shearing features will also maximize 'their efficiency by contacting and subdividing as much food as possible per chewing cycle' (Strait, 1993: 393). Therefore, Strait argued that the most efficient tooth shape to penetrate hard foods has short crest lengths, but for soft foods longer crest lengths are desirable. Her measurements of the length of shearing crests (in the form of 'shearing ratio') of several pairs of 'hard-object' and 'soft-object' feeders showed that 'hard-object' feeders have shorter shearing crests than 'soft-object' feeders.

In this work, we apply and compare the reasoning of Lucas (1982) and Strait (1991) to the problem of how the shape of a tooth affects its efficiency in breaking up 'hard' and 'soft' insects. This was accomplished by conducting experiments involving penetration of punches of different shapes through prey of differing physical properties - prey with a stiff (adult beetle) and pliant (beetle larvae) exoskeleton. This investigation into insect properties differs from most other studies of its type as it concentrates on the fracture properties of the insect as a whole rather than the physical properties of the component parts (see, for example, Hepburn \& Chandler, 1976).

The shape of a tooth or cusp cannot be quantified by a single number or ratio. When considering the force and energy required to penetrate and drive through foods, there are two important aspects of cusp shape that will influence its function. One is the initial surface area of contact between tooth and food. The second is the volume and surface area of the entire tooth. A smaller surface area of contact will increase the stress for a given force; and a smaller tooth volume requires less material to be displaced and probably fewer bonds in the material to be broken for the tooth to be driven through the food. Therefore we will define two aspects of tooth shape/sharpness. 'Tip-sharpness' is the surface area of contact between the tooth and food for a given applied force, which will be proportional to the radius of curvature at the tip (following Lucas, 1982). 'Cuspsharpness' is the volume or surface area of the cusp at increasing distances from the tip. A wedge of, for example, $30^{\circ}$ will have a greater surface area and volume for a given distance from the tip than a wedge of $15^{\circ}$. In this manner, cusp-sharpness is analogous to the included angle of a wedge, but the angle as such cannot be measured on rounded teeth.

These two measures are likely to be relevant in different circumstances. Tip-sharpness will be more pertinent to crack-initiation, as the surface area of contact will determine the pressure applied (for a given force). Cusp-sharpness will be more relevant to crack- propagation. The volume of the tooth will determine the amount of applied energy necessary for the tooth to drive through a non-brittle material, since tooth volume is analogous to the included angle in a wedge. When the wedge angle is large, the energy freed when bonds are broken during fracture is transferred more effectively to the tip of the crack. This energy assists further crackpropagation, so less energy need be applied for this purpose (Vincent, 1990).

The distinction between these two measures, however, becomes blurred for foods of low elastic modulus $(E)$, i.e. pliant foods (Ashby \& Jones, 1980). For a stiff material, the surface area of contact for a given force (i.e. tip-sharpness) of a tooth will be low; however, when the tooth is applied to a pliant material, the corresponding surface area will be greater, as the material will deform around the tooth, resulting in an increase in the surface area of contact. As cusp-sharpness is measured as the volume or surface area at increasing distances from the tip, the measure of tip-sharpness for a tooth will approach that of cusp-sharpness when a pliant material is being considered, as both will take into account the surface area of contact at distances further from the tip.

Obviously, tooth function and the forces involved in the course of normal mastication are not as simple as the experiments carried out here. Processes such as interdigitation of the cusps causing shear or a 'mortar and pestle' effect will be important in the breakdown of foodstuffs. However, the object of this study was not to simulate jaw movement or the chewing cycle, or even entire teeth. This work targets issues of tooth sharpness per se and how they affect fracture rather than attempting to simulate insectivory. Specifically, we were looking for functional differences in tooth shape when the tooth is used to penetrate and drive through insects (presumably most closely analogous to 'puncture-crushing; Hiiemae, 1978), and for the more general consequences of change in tooth shape and the resultant change, if any, in the tooth's effectiveness in dividing foods.

Other than merely testing the effect of varying cuspand tip-sharpness on the punches' ability to divide insects, the punches were designed with two additional factors in mind. The first factor was to test the effect of tooth wear. The punches are members of a series that simulate differing wear states of teeth, i.e. perfectly sharp to progressively blunter ones. The amount of wear that a particular punch has 'undergone' can be shown by the difference in volume in its tip compared to the 'A' punch (see later) of the same angle. This is analogous to the amount of enamel that is removed from teeth during wear. Differences in the forces required to penetrate and drive through material can be interpreted as showing differences in the ability of teeth with different wear states to penetrate insects. The second factor was to test the effect of scale on tooth efficiency. Blunted punches of the same angle are scale diagrams of one another, that is, they are the same shape but at different scales. Differences between scale punches will also show that scale is important. 


\section{MATERIALS AND METHODS}

\section{Design of equipment and punches}

The force necessary for metal punches of various shapes to first pierce and then drive through insects was measured using a modified Chatillon Force Tester (Model UTSE-2). Lateral movement of the driving shaft was reduced by running the shaft through a keyway in a self-lubricating cast iron block. The punch was screwed onto the bottom end of the shaft. Force was applied to the specimen by moving the stage up towards the punch at a constant velocity $(\sim 0.8 \mathrm{~mm} / \mathrm{s})$; the force was measured by the load cell attached to the top end of the shaft, which was connected to a personal computer. This speed was chosen because tests on viscoelastic materials (which include most biological materials) are better carried out at low velocities. Although strictly the results may vary with test velocity, testing at low velocities enhances sensitivity to subtle differences in the fracture properties of the materials (N. Aranwela, G. D. Sanson \& J. Read, unpubl. data). A computer program controlled the collection of force and displacement data at 600 measurements/s (P. Fell, Monash University). Due to the friction produced by the shaft in the keyway of the block, a blank run was taken to record the force produced by the shaft moving in the keyway before each test run. Another computer program (M. Logan, Monash University) was used to align the blank run and test run curves and then subtract the blank from the test run. The subtracted graph can then be used to determine the maximum force and the work to fracture.

The maximum force $(\mathrm{N})$ and the energy $(\mathrm{J})$ necessary to drive the punches through each specimen was recorded from the corrected force $v s$ displacement graphs. Also recorded was the force and energy necessary initially to puncture the cuticle. This was recognizable as the first peak on a force $v s$ displacement curve, i.e. the first local maximum on the curve. Other local maxima may be present on the graph which probably represent the fracture of other components of the insect, or the bursting of the larva in the case of beetle larvae. The force tester was only calibrated up to a force of $50 \mathrm{~N}$, so any force above this was recorded as $50 \mathrm{~N}$.

The punches used to drive through the specimens were based on cones of $30^{\circ}, 60^{\circ}, 90^{\circ}$ and $120^{\circ}$ subtended angles, and 4 punches of each angle were made from high-carbon silver steel. One punch of each angle was left as a pointed cone (termed ' $A$ '). The other 3 were blunted by truncating the cones by 'blunting distances' of $0.5,1.5$ and $3.5 \mathrm{~mm}$ ('B', 'C' and 'D', respectively) from the tip. A curve was then fitted from the centre of the new flattened tip to the 'blunting distance' from the new tip. Curves for punches of the same angle but differing blunting distances were drawn as scale images of one another. This process created 16 punches with a wide range of tip-sharpness and cusp-sharpness from very sharp $\left(30^{\circ} \mathrm{A}\right)$ to very blunt $\left(120^{\circ} \mathrm{D}\right)$ (Fig. 1$)$. A perfectly flat punch was also made (designated $180^{\circ} \mathrm{X}$ ). Scale transparencies of the punches at $\times 10$ magnification were
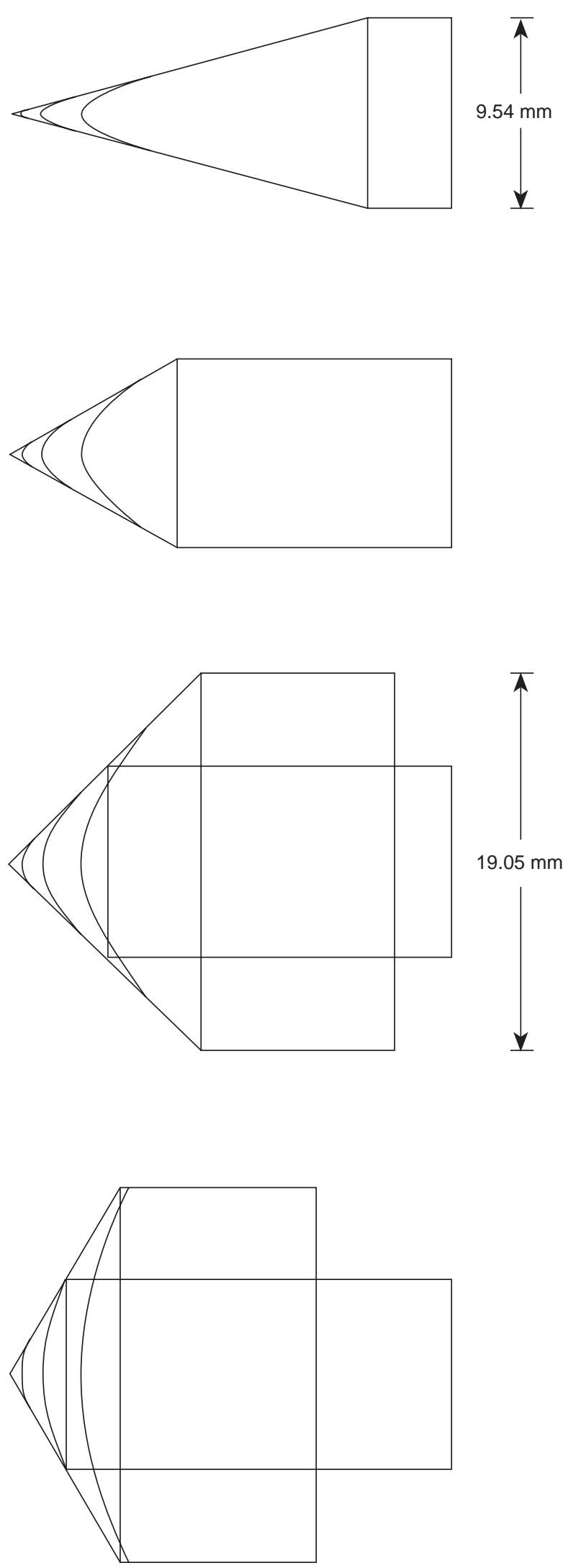

Fig. 1. Scale diagrams of profiles of 16 punches of angles $30^{\circ}$, $60^{\circ}, 90^{\circ}$ and $120^{\circ}$. Punches $30^{\circ} \mathrm{A}-30^{\circ} \mathrm{D}, 60^{\circ} \mathrm{A}-60^{\circ} \mathrm{D}, 90^{\circ} \mathrm{A}-$ $90^{\circ} \mathrm{C}$ and $120^{\circ} \mathrm{A}-120^{\circ} \mathrm{C}$ were constructed from high-carbon silver steel rods of $9.54 \mathrm{~mm}$ diameter; punches $90^{\circ} \mathrm{D}$ and $120^{\circ} \mathrm{D}$ were constructed from rods of $19.05 \mathrm{~mm}$ diameter. 
used on a Nikon V-20A Profile Projector to machine the punches to within approx. $10 \mu \mathrm{m}$ of the desired shape.

\section{Measures of punch shape}

When the force and energy necessary for a tooth to penetrate and drive through prey are considered, the 2 most important aspects of teeth to be measured are 'tipsharpness' and 'cusp-sharpness'. 'Tip-sharpness' of the punches is measured as the radius of curvature of each punch at its tip, and 'cusp-sharpness' as the volume and surface area of the punches per unit distance from the tip.

Radius of curvature, volumes and surface areas cannot be calculated for the $180^{\circ} \mathrm{X}$ punch as these measures would essentially be undefined or infinite for a perfectly flat punch. Therefore, it is excluded from the remainder of the calculations. Radius of curvature cannot be measured for the unblunted ' $\mathrm{A}$ ' punches as the derivative at the point of these punches is undefined.

The radius of curvature of a line is the inverse of the rate of change of curvature with respect to arc length.

$$
\text { Radius of curvature }=\frac{\left(1+f^{\prime}(x)^{2}\right)^{3 / 2}}{f^{\prime \prime}(x)}
$$

The minimum radius of curvature of the punches was found by fitting a parabola to the 'rounded' part of the punch. Scale diagrams of the punches were used to obtain $x, y$ co-ordinates of the rounded tip of the punch. These were then fed into 'Microsoft Excel 5.0' and a polynomial of power 2 was fitted to them. The formula of this parabola was then substituted into the equation for radius of curvature to derive the radius of curvature at the tip of the parabola.

If the volume of a cusp is measured from the tip to a distance $y$ from the tip, and then $y$ is plotted against volume, such a graph will be much steeper for a blunter cusp than a sharper cusp. The same applies for surface area (assuming a relatively smooth surface). Punches 'B' to ' $\mathrm{D}$ ' were modelled by a paraboloid at the tip and a conical section for the rest of the punch. Equations for each of these were used to calculate volume and surface area of the punches as a function of distance from the tip. Volume and surface area of the punches to a distance of $0.5 \mathrm{~mm}$ and $3 \mathrm{~mm}$ from the tip were also calculated. The difference in volume between an untruncated cone ('A') and each of the blunted punches of the same angle was calculated. This is the volume of metal that needs to be removed from the ' $A$ ' punch in order to obtain the other punches, and is analogous to the amount of enamel that is removed in the wear of teeth.

\section{Punching experiments with adult beetles}

Adult and larval Tenebrio sp. beetles were obtained from an insect supplier (Ernst Weiher, Sunbury). They were kept alive until just before the experiments began, when they were placed in a killing jar containing ethyl acetate for 10-15 min. To prevent any lateral or vertical movement of the beetle during the experiment, the ventral surface of each beetle was glued to a thin strip of metal using 'Supa Glue' (cyanoacrylic acid ester), making sure that the legs of the beetle were displaced laterally. The metal strip was then firmly clamped to the stage and arranged so that the punch first contacted each beetle at the centre of the prothorax. Length from anterior to posterior $( \pm 0.01 \mathrm{~mm})$ and weight $( \pm 0.001 \mathrm{~g})$ were measured for each beetle using digital callipers and digital scales, respectively.

Replicates of 15 beetles were taken for each of the 17 punch shapes, and the order of the punches was randomized. The machine was set so that the punch would not contact the metal strip, but stop about $0.2 \mathrm{~mm}$ before touching it. This was to preclude measuring the force once the punch had contacted the metal strip, which would be irrelevant to that needed to drive through the insect, and would damage the strain gauge. The force to drive through to $0.2 \mathrm{~mm}$ will still be referred to as the force necessary to 'drive through' the beetle.

\section{Punching experiments with beetle larvae}

The method for this experiment generally follows that for the beetles, except the larvae were glued to the metal strip using a drop of glue on the ventral surface at both the anterior and posterior ends. The metal strip was clamped so that the punch first contacted the larva at the centre of the third segment after the legs. The length from anterior to posterior $( \pm 0.01 \mathrm{~mm})$ and weight $( \pm 0.0001 \mathrm{~g})$ of each larva were measured.

For each larva, whether the punch pierced the larva, or whether the larva burst or was compressed, was recorded. The larva was considered to have been pierced once the cuticle had been breached at the point of contact between the larva and the punch; a larva had burst when internal fluid exuded from a hole in the cuticle other than one created by direct contact with the punch. This information was then related to the force $v s$ displacement graphs so that the local maxima present on the graphs could be correlated with either the larva being pierced or being burst.

\section{Statistical analyses of punching experiments}

The results for punching experiments were first logged, and then analysed by 1-way ANOVAs for each of the angles with the blunting distance (i.e. tip) as the categorical variable, and then for each tip with the angle as the categorical variable, giving a total of 8 tests for each set of data. As each punch was included in 2 analyses, a Bonferroni adjustment for multiple non-independent tests was made. The $P$-value required for a significant result was $0.05 / 2=0.025$. For the adult beetle data, ANOVAs were carried out on the force to penetrate $(f p)$, energy to penetrate $(e p)$, maximum force $(m f)$ and 
Table 1. Measurements of minimum radius of curvature and volume difference from the 'A' punch of the same angle for the punches used in this study.

\begin{tabular}{lcc}
\hline Punch & $\begin{array}{l}\text { Minimum radius of } \\
\text { curvature }(\mathrm{mm})\end{array}$ & $\begin{array}{l}\text { Volume difference from 'A' } \\
\text { punch }\left(\mathrm{mm}^{3}\right)\end{array}$ \\
\hline $30^{\circ} \mathrm{A}$ & - & 0 \\
$30^{\circ} \mathrm{B}$ & 0.071 & 0.018 \\
$30^{\circ} \mathrm{C}$ & 0.204 & 0.432 \\
$30^{\circ} \mathrm{D}$ & 0.483 & 5.71 \\
$60^{\circ} \mathrm{A}$ & - & 0 \\
$60^{\circ} \mathrm{B}$ & 0.309 & 0.069 \\
$60^{\circ} \mathrm{C}$ & 0.955 & 2.06 \\
$60^{\circ} \mathrm{D}$ & 2.27 & 27.5 \\
$90^{\circ} \mathrm{A}$ & - & 0 \\
$90^{\circ} \mathrm{B}$ & 0.951 & 0.225 \\
$90^{\circ} \mathrm{C}$ & 2.86 & 6.11 \\
$90^{\circ} \mathrm{D}$ & 6.67 & 77.6 \\
$120^{\circ} \mathrm{A}$ & - & 0 \\
$120^{\circ} \mathrm{B}$ & 2.82 & 0.65 \\
$120^{\circ} \mathrm{C}$ & 8.88 & 20.4 \\
$120^{\circ} \mathrm{D}$ & 18.0 & 169 \\
\hline
\end{tabular}

energy to drive through $(e d)$. For the larval data, ANOVAs were carried out only on $m f$ and $e d$, as penetration of the larvae only occurred consistently with 1 punch. Regression analyses were carried out on $f p$ and ep $v s$ the minimum radius of curvature (beetles only), $m f$ and $e d v s$ volume and surface area to both 0.5 $\mathrm{mm}$ and $3 \mathrm{~mm}$ from the tip, and $m f$ and $e d v s$ volume difference from the ' $A$ ' punch of the same angle.

\section{RESULTS}

\section{Design of punches}

Since the minimum radius of curvature of the punches increases with blunting distance within each of the angles (Table 1), the punches will therefore show variation in their initial surface area of contact with the food for a given applied load. Punches of the same blunting distance but of a smaller angle have smaller radii of curvature than those of a larger angle. This shows that decreased tip-sharpness is likely to correlate with decreased cusp-sharpness.

Punches of greater angles have greater volume and surface area at increasing distances from the tip (Fig. 2), and therefore have lower cusp-sharpness. Within each angle, those with a larger blunting distance have a lower cusp-sharpness. The volume difference of the blunted punches from the ' $A$ ' punch increases as blunting distance increases for each of the angles (Table 1).

\section{Punching experiments with adult beetles}

The results of experiments measuring force and energy needed to initially puncture the exoskeleton of the beetle can be summarized as follows (Fig. 3a shows the results for force measurements; for all experiments, the results
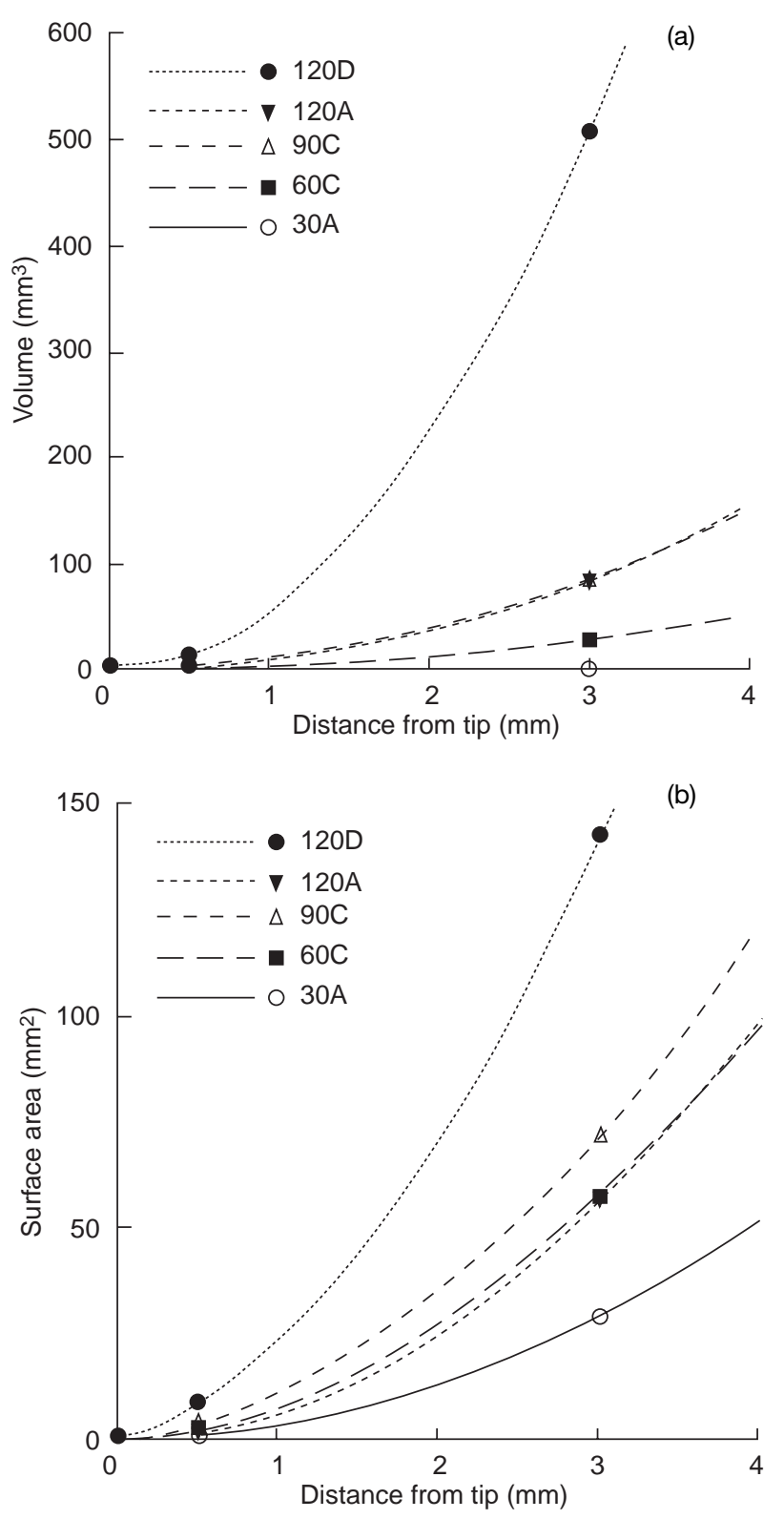

Fig. 2. (a) Volume and (b) surface area of five of the punches used in this study plotted against distance from tip. 30, 60, 90 and 120, the angle (degrees) of the punch; A, C and D, the blunting distance of $0.0,1.5$ and $3.5 \mathrm{~mm}$, respectively.

for energy measurements follow the same pattern as for those of force):

(a) for punches of the same blunting distance (e.g. $30^{\circ} \mathrm{B}$ and $60^{\circ} \mathrm{B}$ ), those of a larger angle required significantly more force and energy to penetrate than those of a smaller angle $(P<0.001$ for all analyses).

(b) for punches of the same angle (e.g. $30^{\circ} \mathrm{B}$ and $30^{\circ} \mathrm{C}$ ), those with a greater blunting distance generally required more force and energy. Significant differences were found between punches of the same angle for all except the $30^{\circ}$ punches (for $30^{\circ}$ punches, force to penetrate, d.f. $=3, F=2.39, P=0.078$, and energy to penetrate, d.f. $=3, \quad F=2.59, \quad P=0.062 ; \quad P<0.001$ for all other 

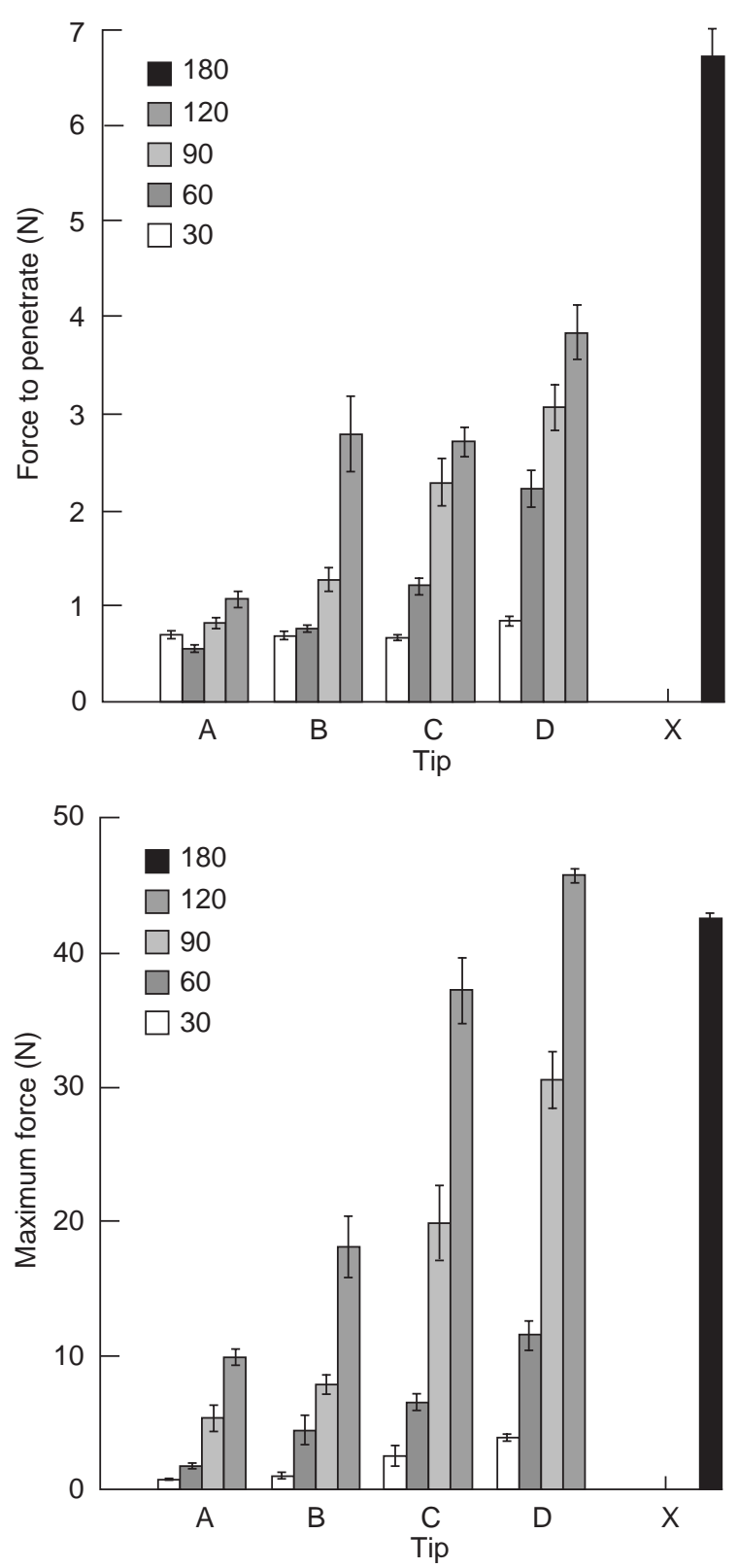

Fig. 3. Results of punching adult beetles with punches of different shape: (a) force to penetrate (mean $\pm \mathrm{SE}$ ) plotted against punch; (b) maximum force (mean $\pm \mathrm{SE}$ ) plotted against punch. 30, 60, 90 and 120, the angle (degrees) of the punch; A, $\mathrm{B}, \mathrm{C}$ and $\mathrm{D}$, the blunting distance of $0.0,0.5,1.5$ and $3.5 \mathrm{~mm}$, respectively; $180 \mathrm{X}$, a flat punch.

angles). This shows that teeth of the same shape but at different scales require different forces to penetrate.

When the force and energy required to penetrate the beetle are plotted against minimum radius of curvature (Fig. 4), punches with a smaller minimum radius of curvature required less force and energy to penetrate the beetle. The slope of the regression line for all of the punches is significantly different from zero (for force, $r=0.698, F=168.86, P<0.001 ;$ for energy, $r=0.42$, $F=38.03, P<0.001)$. The regression slopes for each of the four angles tend to decrease for increasing angles.

(a)

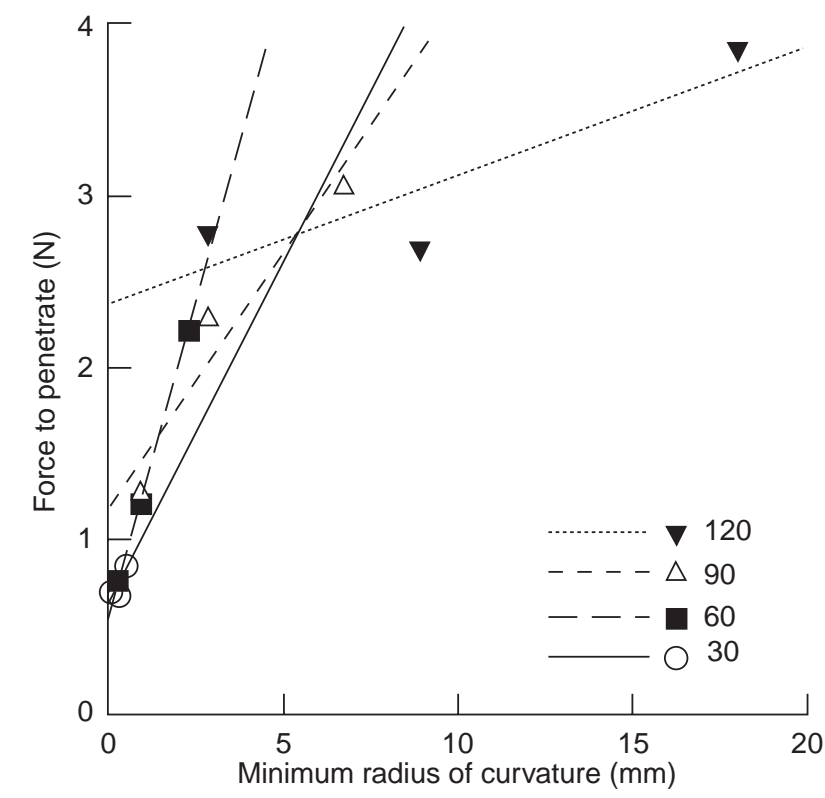

Fig. 4. Force (mean $\pm \mathrm{SE}$ ) required to penetrate beetles plotted against minimum radius of curvature of the punch. 30, 60, 90 and 120, the angle (degrees) of the punch used.

The results for the maximum force and energy needed to drive through beetles are (see Fig. 3b):

(a) for punches with the same blunting distance, those with the greater subtended angle require greater force and work than those with a smaller angle. Significant differences were found between punches of the same blunting distance $(P<0.001$ for all analyses).

(b) for punches of the same angle, those with a smaller blunting distance required less force and work. Significant differences were found between punches of the same angle but different blunting distance $(P<0.001$ for all analyses).

Punches with a greater volume to $0.5 \mathrm{~mm}$ and $3 \mathrm{~mm}$ from the tip required more force and energy to drive through the beetles $(P<0.001$ for all analyses $)$. The slope of the regression line for the four angles tended to decrease at increasing angles. Correlations between surface area to $0.5 \mathrm{~mm}$ and $3 \mathrm{~mm}$ and maximum force and energy are also significant $(P<0.01)$. The slope of the regression lines for the four angles tends to increase with increasing angle.

Punches with greater volume difference to the ' $\mathrm{A}$ ' punch of the same angle required more force and energy to drive through the beetles $(P<0.001$ for all analyses; Fig. 5). The slope of the regression lines decrease with increasing angle.

\section{Punching experiments with beetle larvae}

Only the sharpest punch, $30^{\circ} \mathrm{A}$, was able to consistently penetrate the larvae ( $93 \%$ of trials, $n=15)$, and so the force and energy required to actually penetrate the larvae can only be measured for this punch (mean $\pm \mathrm{SE}$, 


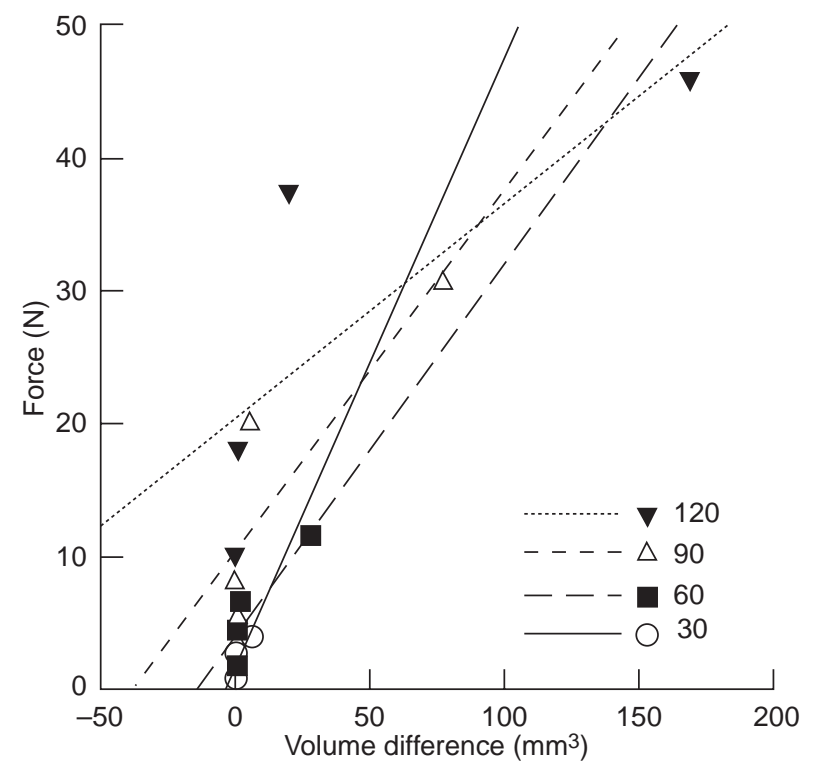

Fig. 5. Force (mean $\pm S E$ ) necessary to drive through beetles plotted against volume difference from the ' $A$ ' punch of the same angle. 30, 60, 90 and 120, the angle (degrees) of the punch used.

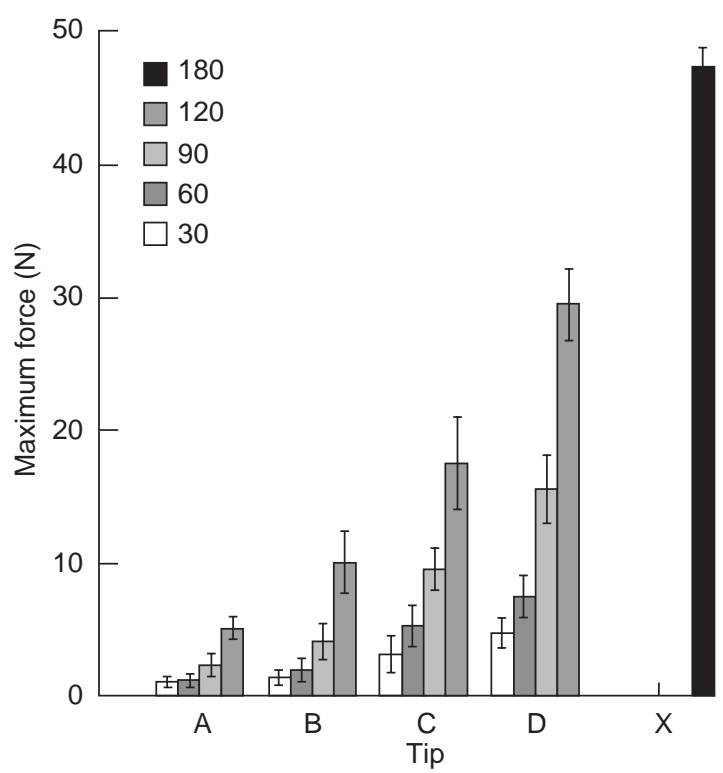

Fig. 6. Results of punching beetle larvae with punches of different shape. Maximum force (mean $\pm \mathrm{SE}$ ) plotted against punch. 30, 60, 90 and 120, the angle of the punch used; A, B, C and $\mathrm{D}$, the blunting distance of $0.0,0.5,1.5$ and $3.5 \mathrm{~mm}$, respectively; 180X, a flat punch.

$\left.0.29 \pm 0.084 \mathrm{~N}, 9.4 \times 10^{-5} \pm 7.1 \times 10^{-5} \mathrm{~J}\right)$. For the majority of the other punches, the force and energy recorded was that needed to compress the specimen (Fig. 6):

(a) for punches with the same blunting distance, those of a larger angle required greater force and energy $(P<0.001$ for all analyses $)$. (b) for punches of the same angle, those of a larger blunting distance required greater force and energy $(P<0.01$ for all analyses).

Punches with a greater volume to $0.5 \mathrm{~mm}$ and $3 \mathrm{~mm}$ from the tip required greater force and energy to drive through or compress the larvae $(P<0.001$ for all analyses). The slope of the regression lines for each of the angles tended to decrease for increasing angle. Correlations between surface area to $0.5 \mathrm{~mm}$ and $3 \mathrm{~mm}$ from the tip and maximum force and energy were also significant $(P<0.01)$. The slope of the regression lines for each of the angles increases for increasing angle.

Punches with a greater difference in volume from the 'A' punch required more force and energy to drive through or compress the larvae $(P<0.001$ for all analyses). The slope of the regression lines for each of the angles tended to decrease for increasing angle.

\section{DISCUSSION}

It is clear from the results that tooth structure can greatly influence the force required to break up insects. The force and energy necessary to penetrate the insects were proportional to the surface area of contact, i.e. the radius of curvature. This follows the prediction of Lucas (1982) that teeth should possess the smallest radius of curvature for the dental material involved, as the force required to penetrate will decrease with decreasing radius of curvature. Lucas (1982) predicts that harder, more brittle foods can be reduced equally effectively by blunt cusps, which reduces the risk of tooth tip fracture. He states that a greater radius of curvature can be tolerated for harder foods for initial penetration as the surface area of contact between (a) a pliant object and a sharp tooth and (b) a stiff object and a blunt tooth will be approximately the same. This does not apply, however, when considering the further penetration of the tooth into tough materials and structures, such as insects. Insects are not homogeneously brittle, and, as our experiments found, they do not completely fracture upon the application of sufficient force. The experiments above showed that an increase in tooth volume meant more force and energy were required to drive through insect prey. This means that insects cannot be reduced equally effectively by blunter teeth (those with a lower cusp-sharpness) due to the additional force needed to drive through the internal tissues of the insect. It is, therefore, important to measure tooth volume (cuspsharpness) as well as tip-sharpness as both will have an effect on the necessary force and energy to be applied by the tooth.

Strait $(1991,1993)$ suggested that 'blades' used to divide stiff, tough materials should be as short as possible in order to reduce the surface area of contact between the blades and the food (Fig. 7a). She considered these 'blades' to be analogous to the crests on teeth, and so she argues that the crests on teeth should be as short as possible. However, the tribosphenic (trituberculate) teeth of insectivores are not structured 

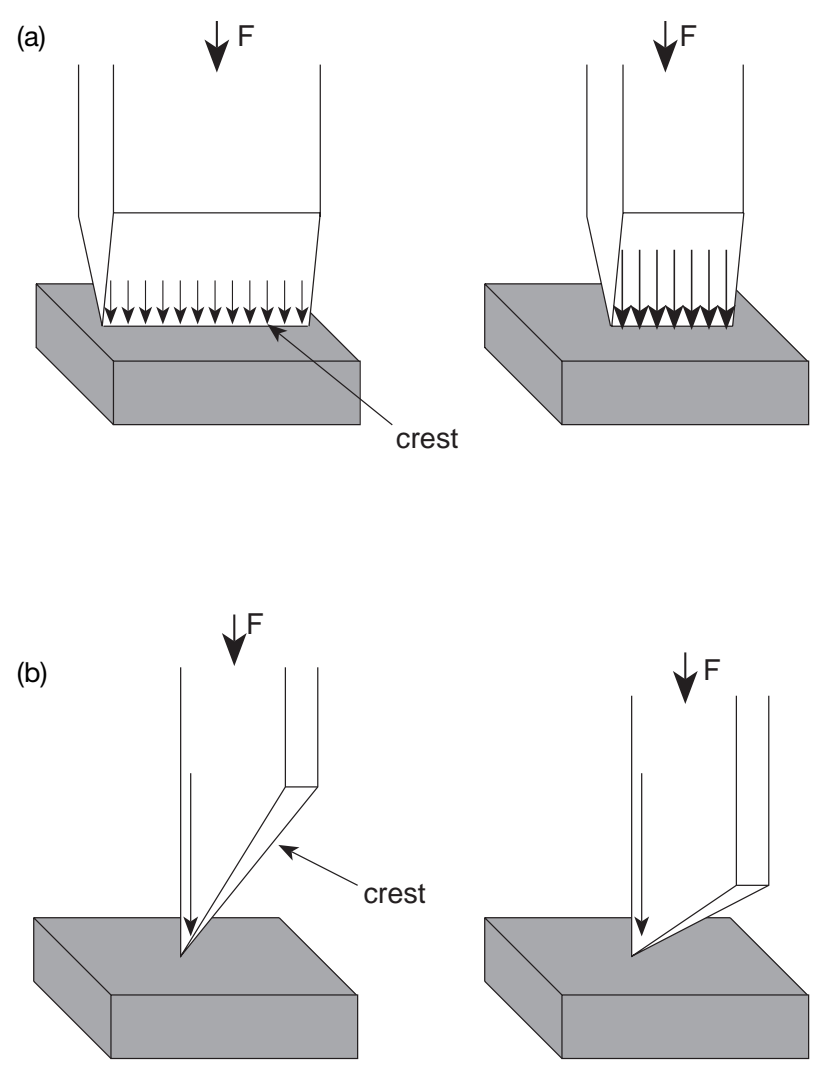

Fig. 7. Relationship between crest length and surface area of contact. The relative lengths of the small arrows represent the pressure magnitudes. Given an applied load of equal force $(F)$, the smaller the area of initial contact the greater the pressure. (a) Strait's (1991, 1993) interpretation, where a longer crest length (left) has a greater surface area of contact than a shorter crest length (right). (b) The interpretation preferred in this work, where the crest is angled away from the prey. A longer crest length (left) has the same surface area of contact as a shorter crest length (right).

in this manner. In fact, the crest is angled away from the food (Fig. 7b), not parallel to it as implied by Strait. The result is that the whole length of the crest does not contact the food at one time. Therefore, the length of the shearing crest will not in itself be the main factor in estimating the surface area of contact; only the surface area of the cusp tip will be important, or that part of the blade in contact at any one moment.

The logic used by Strait in reaching the conclusion that shorter crest lengths are the optimal tooth shape for breaking up stiff-shelled insects therefore does not follow. For a given basal cusp area, a tooth with a greater crest length will generally have a greater height accordingly (Fig. 8). It will also decrease the included angle and so increase its cusp-sharpness. Teeth of higher cuspsharpness will generally have a higher tip-sharpness, as shown by the punches in this study. Hence, a tooth with a longer crest length will require less force to penetrate an object than one with a shorter crest length because it will have greater tip- and cusp-sharpness. Rather than being

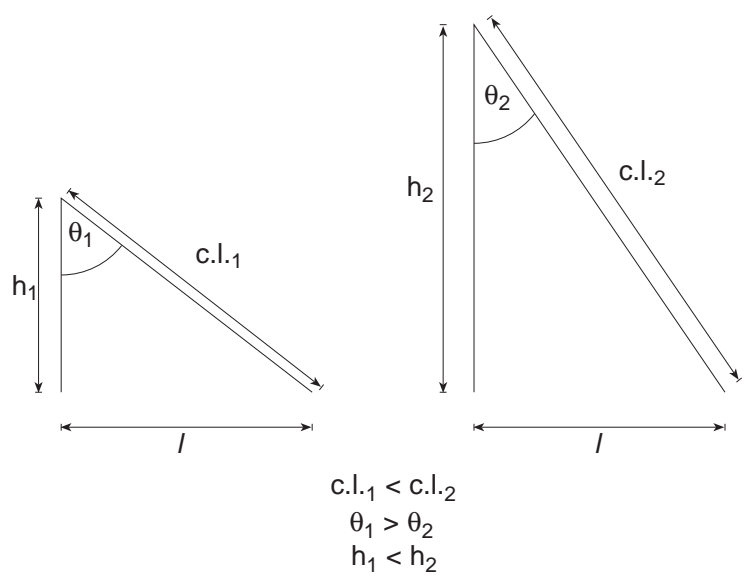

Fig. 8. Relationship between crest length and included angle $\theta$ for a given tooth of length $l$; left, cusp with shorter crest length (c.1.1), which has a larger angle $\left(\theta_{1}\right)$ and is taller than cusp on right, with longer crest length (c.1.2); c.l., crest length; h, tooth height.

merely tolerated, a long crest length on a tribosphenic tooth will, in fact, be of great advantage in penetrating and driving through both pliant and stiff prey.

In addition, the penetration of pliant prey will actually be more easily achieved by a cusp than a blade. The crest of the cusp acts as a 'blade' to divide the material once it has been penetrated by the tip. This is analogous to using the point of a knife to penetrate a tomato before dividing it completely using the blade. A triangular cusp compared to a circular one will also be better in the penetration of ductile objects, as observed by the use of triangular needles by surgeons to pierce the skin (Freeman, 1979). 'Cutting' of viscoelastic materials along the crests in the manner of a guillotine will also be more efficient for longer crests. A cusp with a longer crest length is likely to have a smaller angle with respect to the vertical, and as the mechanical advantage of this system is $1 / \sin (\theta)$, less force is required for such a crest to cut the material.

Strait (1991: 148-151, 1993: 393) also hypothesized that long crests will maximize 'efficiency by contacting and subdividing as much food per chewing cycle'. This is not necessarily so. If the crest length is increased without an increase in the horizontal tooth length, the amount of material that could be divided with one stroke is not increased. The amount of material divided depends on the horizontal length of the crest, not its length in three dimensions.

What, then, does the 'shearing ratio' actually measure? In most cases it indirectly measures the height of the cusps. A number of authors have found taller cusps to be advantageous in reducing prey with a ductile exoskeleton. Wright (1988) found that beetle larvae could not be as finely reduced by Isoodon macrourus as by Dasyurus hallucatus, which has higher, sharper cusps. The remains of the larvae in Isoodon were compressed but not cut (Wright, 1988). Similarly, Moore \& Sanson (1995) found that the grinding bunodont molars of 
Petaurus breviceps were less efficient at removing nitrogen from larval insects than Dasyuroides byrnei with a tribosphenic dentition capable of shear.

In many studies on the physical properties of insects as prey, often only one component of the insect has been considered. Most commonly this has been the cuticle, described as hard, soft, stiff, brittle, etc. The above experiments have shown that in order to divide the insect, not only the exoskeleton needs to be penetrated, but also the internal tissues. Additional force and energy are required to do this, as shown by the difference in force required to drive through the insects compared with that needed merely to penetrate them. This is contradictory to the implicit view previously held, as shown by Strait's diagram of a beetle 'shattering' once it has been penetrated (Strait, 1993: fig. 1). As a tooth penetrates the prey, all internal organs need to be fractured independently. The experiments show us that different insects do fail differently, and require different forces to penetrate and drive through.

Strait found a difference in tooth shape between the teeth of hard- and soft-object feeders, even though these experiments showed that the most efficient tooth shape for penetrating and driving through hard and soft insects was the same. The difference is likely to be due to various compromises between functions that the teeth must perform, and the physical constraints on them. The risk of tooth tip fracture has been considered a significant restriction on sharpness (Lucas, 1982; Hill, 1985; Strait, 1993); animals may have evolved blunter teeth to reduce the risk of tooth fracture when eating harder foods. For insectivores, at least, this risk may not be as high as previously thought, since no fractured teeth were noted on 38 specimens of insectivores (A. R. Evans, pers. obs.). A likely explanation lies in the conflicting functions of teeth (Wright, 1988; Moore \& Sanson, 1995; Evans, 1996). For example, it has been found that the teeth of hard-object consumers have a better-developed grinding area on their teeth, presumably for the reduction of the brittle exocuticle. This has lead to a decreased height of the cusps, and thereby a reduction in the sharpness of the teeth. The conflict between the need for a grinding basin and the greater efficiency of taller, sharper teeth has resulted in the sharpness of the teeth being compromised. Further investigations should focus on differences between hardand soft-object feeders in terms of differences in tip- and cusp-sharpness, and on the determination of exactly which compromises or constraints are acting on the teeth of insectivores.

Other aspects of tooth and cusp shape were not investigated in this study, such as the effect of differences in the overall shape of teeth (i.e. other than a conical tooth shape), the presence of crests, and the sharpness of these crests. The shape of the cusp will be important in the breakdown of foods, e.g. as Freeman (1992) showed with regards to the assistance of crackpropagation by 'crests' on the canines of certain bats. The finding of increased efficiency of cusps of smaller radius of curvature and volume in these experiments, however, should have general applicability when comparing teeth of a similar overall structure.

The design of the punches allows us to draw conclusions on two varied aspects of tooth form: tooth wear and scale. Wear on teeth during the lifetime of an animal will have a large effect on the efficiency of those teeth. The teeth of certain mammals (such as humans: Luke \& Lucas, 1983) are fully functional upon eruption, unlike those of many herbivorous forms (Janis \& Fortelius, 1988). In these cases, the tooth presumably has its maximum efficiency as soon as it has taken its place in occlusion in the tooth row. Therefore, no prior wear is necessary for the proper and efficient functioning of these teeth, and any wear will most likely decrease the tooth's efficiency. If animals were born with teeth the sharpness of the ' $A$ ' punches in these experiments, and the tips of teeth wear down in a simple fashion to resemble the ' $D$ ' punches, then worn teeth will be much less effective in penetrating and driving through foods as they will require greater force and energy to divide foods (Fig. 5). Several authors have studied wear on teeth and the associated changes in efficiency (e.g. Every \& Kuhne, 1971; Rensberger, 1973; Lanyon \& Sanson, 1986), and have found that in some cases self-sharpening mechanisms have been used. These mechanisms presumably maintain the tooth's high tip-sharpness (low radius of curvature at the tip) and high cusp-sharpness (low cusp volume). This maintenance may in part be due to factors such as the detailed structure of the enamel, including enamel prism direction and planes of enamel cleavage, leading to enamel wear that is more likely to produce a sharp rather than a blunt tooth. An analysis of these factors may then show that the tooth shape produced by wear is 'pre-determined' by the structure of the enamel. Another hypothesis for teeth maintaining their high tip- and cusp-sharpness is the relative levels of attrition and abrasion on the teeth (Rensberger, 1973, 1978). Further investigation into the changes in tooth shape and the organization of enamel would support or nullify these hypotheses.

Animals of different sizes are often scaled to a common feature, such as body mass. Measurements of the physical features are also often taken as ratios of a body part and some measure of body size, or the ratio of two different body parts, making the measurements independent of size. A common use of this technique is the calculation of the mechanical advantage of limbs of different-sized animals. Mechanical advantage is a ratio, and is therefore scale independent. However, the same cannot be said for teeth. Tooth function is scale dependent. Teeth of the same shape but different scales will require different forces to penetrate foods, as shown by the punching experiments. This difference in tooth function between animals of different sizes does not seem to have been considered in previous studies, even though many studies have analysed function on scaled teeth (e.g. Kay, 1975; Strait, 1991). The justification of comparing teeth scaled to body size can then be questioned, as in fact the efficiencies of these teeth will be a function of their original size. This study indicates that teeth need 
to be studied at their original scale in order for their actual efficiency to be ascertained and for efficiencies and functions to be compared.

\section{Acknowledgements}

We thank Pat and Ed Grey, and Gudrun Arnold for reading drafts of this paper, and for their helpful suggestions. Nuvan Aranwela was very obliging in clarifying certain materials engineering aspects for us. Thanks are also due to Murray Logan and Peter Fell for designing essential computer programs, Ernst Weiher for the supply of insects, and Peter Domelow and Ian Stewart for their help in the manufacture of the punches.

\section{REFERENCES}

Ashby, M. F. \& Jones, D. R. H. (1980). Engineering materials 1: an introduction to their properties and applications. Oxford: Pergamon Press.

Black, H. L. (1974). A North temperate bat community: structure and prey populations. J. Mammal. 55: 138-157.

Evans, A. R. (1996). Dietary properties and tooth form in insectivores. B.Sc.(Hons) thesis, Monash University, Australia.

Every, R. G. \& Kuhne, W. G. (1971). Bimodal wear of mammalian teeth. In Early mammals: 23-26. Kermack, D. M. \& Kermack, K. A. (Eds). Zool. J. Linn. Soc. 50 suppl. 1.

Freeman, P. W. (1979). Specialized insectivory: beetle-eating and moth-eating molossid bats. J. Mammal. 60: 467-479.

Freeman, P. W. (1981). Correspondence of food habits and morphology in insectivorous bats. J. Mammal. 62: 166-173.

Freeman, P. W. (1992). Canine teeth of bats (Microchiroptera): size, shape and role in crack-propagation. Biol. J. Linn. Soc. 45 : 97-115.

Gordon, J. E. (1968). The new science of strong materials, or why you don't fall through the floor. Harmondsworth: Penguin.

Hepburn, H. R. \& Chandler, H. D. (1976). Material properties of arthropod cuticles: the arthrodial membranes. J. comp. Physiol. 109: $177-198$.

Hiiemae, K. M. (1978). Mammalian mastication: a review of the activity of the jaw muscles and the movements they produce in chewing. In Development, function and evolution of teeth: 359 398. Butler, P. M. \& Joysey, K. A. (Eds). London: Academic Press.
Hill, D. (1985). Functional dental morphology in some small dasyurids. B.Sc.(Hons) thesis, Monash University, Australia.

Janis, C. M. \& Fortelius, M. (1988). On the means whereby mammals achieve increased functional durability of their dentitions, with special reference to limiting factors. Biol. Rev. 63 : 197-230.

Kay, R. F. (1975). The functional adaptations of primate molar teeth. Am. J. Phys. Anthropol. 43: 195-216.

Kay, R. F. \& Hiiemae, K. M. (1974). Jaw movement and tooth use in recent fossil primates. Am. J. Phys. Anthropol. 40: $227-$ 256.

Lanyon, J. M. \& Sanson, G. D. (1986). Koala (Phascolarctos cinereus) dentition and nutrition II. Implications of tooth wear in nutrition. J. Zool. (Lond.) 209: 169-181.

Lucas, P. W. (1979). The dental-dietary adaptations of mammals. N. Jb. Geol. Paläont. Mh. 8: 486-512.

Lucas, P. W. (1982). Basic principles of tooth design. In Teeth: form, function and evolution: 154-162. Kurtén, B. (Ed.). New York: Columbia University Press.

Lucas, P. W. \& Luke, D. A. (1984). Chewing it over: basic principles of food breakdown. In Food acquisition and processing in primates: 283-301. Chivers, D. J., Wood, B. A. \& Bilsborough, A. (Eds). New York: Plenum Press.

Luke, D. A. \& Lucas, P. W. (1983). The significance of cusps. J. Oral Rehab. 10: 197-206.

Moore, S. J. \& Sanson, G. D. (1995). A comparison of the molar efficiency of two insect-eating mammals. J. Zool. (Lond.) 235 : 175-192.

Rensberger, J. M. (1973). An occlusal model for mastication and dental wear in herbivorous mammals. J. Paleontol. 47: 515528.

Rensberger, J. M. (1978). Scanning electron microscopy of wear and occlusal events in some small herbivores. In Development, function and evolution of teeth: 415-438. Butler, P. M. \& Joysey, K. A. (Eds). London: Academic Press.

Ross, A. (1967). Ecological aspects of the food habits of insectivorous bats. Proc. West. Found. Vertebr. Zool. 1: 205-264.

Strait, S. G. (1991). Dietary reconstruction in small-bodied fossil primates. PhD dissertation, State University of New York, Stony Brook.

Strait, S. G. (1993). Molar morphology and food texture among small-bodied insectivorous mammals. J. Mammal. 74: 391-402.

Vincent, J. F. V. (1990). Fracture properties of plants. Adv. Bot. Res. 17: 235-287.

Warner, R. M. (1985). Interspecific and temporal dietary variation in an Arizona bat community. J. Mammal. 66: 45-51.

Wright, W. (1988). Functional molar morphology in four genera of marsupial bandicoots. B.Sc.(Hons) thesis, Monash University, Australia. 